\title{
A MECHANISM FOR STIMULATING AGN FEEDBACK BY LIFTING GAS IN MASSIVE GALAXIES
}

\author{
B. R. McNamara ${ }^{1,2}$, H. R. Russell ${ }^{3}$, P. E. J. $\operatorname{Nulsen}^{4,5}$, M. T. Hogan ${ }^{1,2}$, A. C. Fabian ${ }^{3}$, F. Pulido ${ }^{1}$, and A. C. Edge ${ }^{6}$ \\ ${ }^{1}$ Department of Physics and Astronomy, University of Waterloo, 200 University Avenue West, Waterloo, ON N2L 3G1, Canada \\ ${ }^{2}$ Perimeter Institute for Theoretical Physics, Waterloo, ON N2L 2Y5, Canada \\ ${ }^{3}$ Institute of Astronomy, Madingley Road, Cambridge CB3 OHA, UK \\ ${ }_{5}^{4}$ Harvard-Smithsonian Center for Astrophysics, 60 Garden Street, Cambridge, MA 02138, USA \\ ${ }^{5}$ ICRAR, University of Western Australia, 35 Stirling Highway, Crawley, WA 6009, Australia \\ ${ }^{6}$ Centre for Extragalactic Astronomy, Department of Physics, Durham University, Durham DH1 3LE, UK \\ Received 2016 April 13; revised 2016 June 29; accepted 2016 July 20; published 2016 October 14
}

\begin{abstract}
Observation shows that nebular emission, molecular gas, and young stars in giant galaxies are associated with rising X-ray bubbles inflated by radio jets launched from nuclear black holes. We propose a model where molecular clouds condense from low-entropy gas caught in the updraft of rising X-ray bubbles. The low-entropy gas becomes thermally unstable when it is lifted to an altitude where its cooling time is shorter than the time required to fall to its equilibrium location in the galaxy, i.e., $t_{\mathrm{c}} / t_{\mathrm{I}} \lesssim 1$. The infall speed of a cloud is bounded by the lesser of its free-fall and terminal speeds, so that the infall time here can exceed the free-fall time by a significant factor. This mechanism is motivated by Atacama Large Millimeter Array observations revealing molecular clouds lying in the wakes of rising X-ray bubbles with velocities well below their free-fall speeds. Our mechanism would provide cold gas needed to fuel a feedback loop while stabilizing the atmosphere on larger scales. The observed cooling time threshold of $\sim 5 \times 10^{8} \mathrm{yr}$-the clear-cut signature of thermal instability and the onset of nebular emission and star formation-may result from the limited ability of radio bubbles to lift low-entropy gas to altitudes where thermal instabilities can ensue. Outflowing molecular clouds are unlikely to escape, but instead return to the central galaxy in a circulating flow. We contrast our mechanism to precipitation models where the minimum value of $t_{\mathrm{c}} / t_{\mathrm{ff}} \lesssim 10$ triggers thermal instability, which we find to be inconsistent with observation.
\end{abstract}

Key words: galaxies: clusters: general - galaxies: evolution - galaxies: individual (M87, MS 0735+7421, Abell 2029) - X-rays: galaxies: clusters

\section{INTRODUCTION}

Energetic feedback from nuclear black holes is thought to regulate the growth of massive galaxies from their nascency during the quasar era (Fabian 2012) through to their maturity as radio galaxies (McNamara \& Nulsen 2007, 2012). Atomic and molecular outflows observed in quasars and active galaxies apparently regulate star formation and, in some instances, sweep the host galaxy of its gas during the most active phases of galaxy growth (e.g., Morganti et al. 2005; Arav et al. 2008; Nesvadba et al. 2008; Alexander et al. 2010; Feruglio et al. 2010). Mrk 231, for example, has revealed several $\times 10^{8} M_{\odot}$ of molecular gas flowing out of its inner $1 \mathrm{kpc}$ or so with velocities exceeding $700 \mathrm{~km} \mathrm{~s}^{-1}$ (Rupke et al. 2007). The evolution of giant ellipticals at late times is governed instead by radio jets that heat the hot, $\mathrm{X}$-ray atmospheres of galaxies and clusters that would otherwise cool and sustain star formation (Bîrzan et al. 2004; Best et al. 2007; Dunn \& Fabian 2008; Rafferty et al. 2008). Archetypes include NGC 1275 in Perseus (Fabian \& Sanders 2007), M87 (Forman et al. 2007, 2016), and the normal giant elliptical galaxies M84 (Finoguenov \& Jones 2001) and NGC 5813 (Randall et al. 2015). This socalled radio mode or radio-mechanical feedback is responsible in whole or in part for the inefficiency of star formation in the central galaxies of massive halos as they age, leaving them red and dead (Bower et al. 2006; Croton et al. 2006).

New observations of molecular gas in central galaxies made with the Atacama Large Millimeter Array (ALMA) and new numerical simulations of radio bubbles rising in hot atmospheres suggest a richer, more complex picture. Following on discoveries of upward of $10^{9} M_{\odot}$ of molecular gas in central cluster galaxies (Edge 2001; Salomé \& Combes 2003), ALMA and IRAM observations of a half dozen or so central galaxies, including NGC 1275 (Salomé et al. 2006, 2008a, 2008b, 2011), Abell 1835 (McNamara et al. 2014), NGC 5044 (David et al. 2014), and PKS 0745-191 (Russell et al. 2016), indicate that molecular clouds are either lifted out by or condensing along the trajectories of buoyantly rising X-ray bubbles inflated by radio jets. Furthermore, the molecular clouds are moving at surprisingly slow speeds with respect to the velocity dispersion of the stars (Russell et al. 2016) and well below the escape speed of the central galaxy. Observation indicates the molecular clouds are circulating in the potential well of the galaxy while fueling star formation at rates of several to several tens of solar masses per year (Salomé et al. 2011; McNamara et al. 2014).

Star formation and associated nebular emission are hallmarks of galaxies and clusters hosting hot atmospheres with cool cores (Johnstone et al. 1987; Heckman et al. 1989). Chandra X-ray observations have established a cooling time threshold in these systems for the onset of nebular emission and star formation. Star formation and nebular emission are prevalent when the central atmospheric cooling time falls below $\sim 5 \times 10^{8} \mathrm{yr}$ or, similarly, the central entropy parameter falls below $30 \mathrm{keV} \mathrm{cm}^{2}$ (Cavagnolo et al. 2008; Rafferty et al. 2008). Systems lying above the central cooling time threshold are usually devoid of cooling gas and star formation, while those below usually are not. The threshold may be related to the onset of thermal instability in the hot atmosphere (Voit et al. 2008), but the reasons for its numerical value are not understood. 

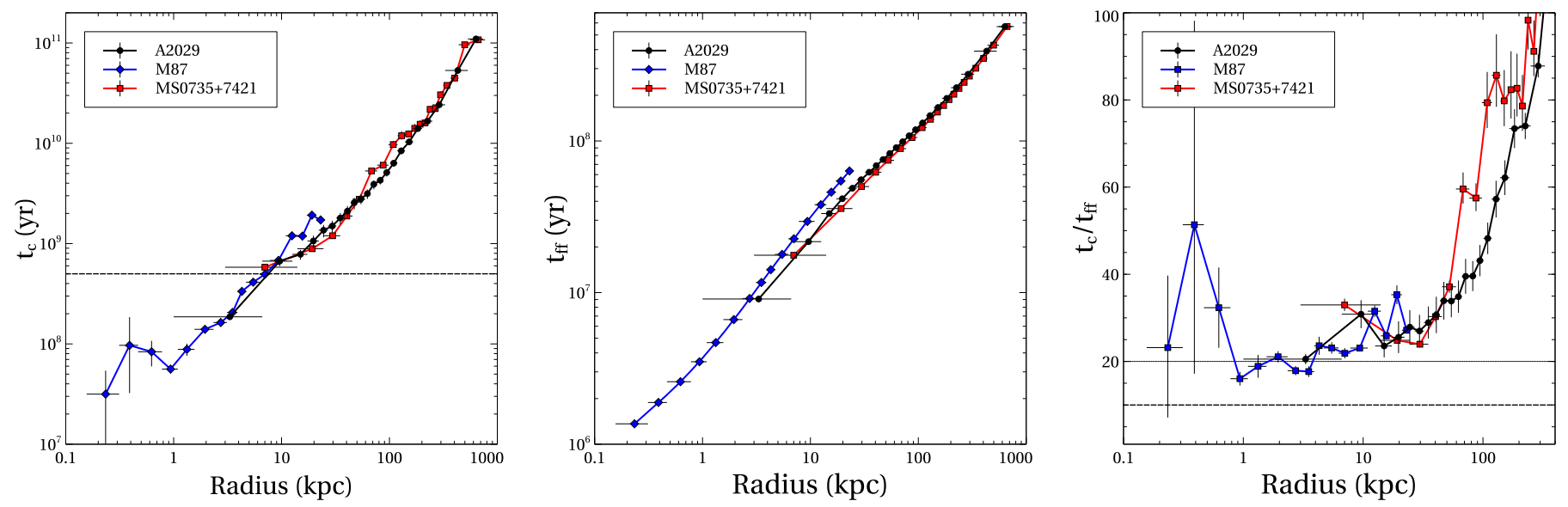

Figure 1. Radial run of cooling time calculated using deprojected gas densities, free-fall times, and the ratio of these quantities.

Theoretical studies of thermal instabilities in cluster atmospheres have attributed the cooling time threshold to thermal conduction, which tends to stabilize cooling atmospheres, and to the ratio of the local cooling time to free-fall time for thermally unstable clouds (Gaspari et al. 2012; McCourt et al. 2012; Sharma et al. 2012; Voit et al. 2015). These studies concluded that when the ratio of the cooling time to free-fall time falls below $t_{\mathrm{c}} / t_{\mathrm{ff}} \lesssim 10$, thermal instability ensues, fueling nebular emission and star formation. While some systems are consistent with this criterion (McCourt et al. 2012; Voit \& Donahue 2015; Voit et al. 2015; Loubser et al. 2016), we show here that the observed values of this ratio are governed almost entirely by the cooling time (the numerator), not the free-fall time. Furthermore, the criterion as applied in these studies forecasts $\mathrm{H} \alpha$ emission less reliably than the central cooling time or central entropy alone.

Motivated primarily by new ALMA observations, we suggest instead that thermal instabilities occur preferentially when cool, X-ray-emitting gas lying within the central galaxy is lifted to higher altitudes behind buoyant X-ray bubbles inflated by radio active galactic nuclei (AGNs). This effectively increases the infall time of the gas, promoting condensation into molecular clouds in the bubbles' wakes. The surprisingly slow molecular cloud velocities found by ALMA (McNamara et al. 2014; Russell et al. 2016) indicate that the infall timescale $\left(t_{\mathrm{I}}\right)$ is substantially longer than the free-fall timescale, promoting thermal instability. In this new picture of feedback, rising X-ray bubbles responsible for heating hot atmospheres and regulating cooling and star formation simultaneously promote cooling in their wakes, fueling an ongoing feedback loop in a mechanism we refer to as stimulated feedback.

\section{THE ONSET OF NEBULAR EMISSION AND STAR FORMATION IN CENTRAL GALAXIES}

Despite a common misperception that central cluster galaxies are dormant, nebular emission, star formation, and other indications of cooling gas are common in cool core or cooling flow clusters (Cowie et al. 1983; Hu et al. 1985; Heckman et al. 1989). The close association between cooling atmospheres and star formation directly links the growth of central galaxies and their nuclear black holes to the reservoir of hot gas surrounding them. Chandra X-ray images have revealed thermodynamic thresholds indicating that nebular emission and star formation ensue when a hot atmosphere's central cooling time and entropy index fall below $t_{\mathrm{c}} \lesssim 5 \times 10^{8} \mathrm{yr}$ and $K \lesssim 30 \mathrm{kev} \mathrm{cm}^{2}$, respectively (Cavagnolo et al. 2008; Rafferty et al. 2008). Galaxies hosting atmospheres lying above these thresholds do not shine with nebular emission or star formation, while those lying below usually do. The thresholds are remarkably sharp, indicating a direct connection between galaxy evolution, feedback, and atmospheric cooling. The thresholds forecast $\mathrm{H} \alpha$ emission more reliably than star formation, most likely because small levels of molecular and atomic gas $\left(\lesssim 10^{6} M_{\odot}\right)$ emit detectable levels of $\mathrm{H} \alpha$ emission before the galaxy has accumulated enough molecular gas $\left(\sim 10^{9} M_{\odot}\right)$ to fuel appreciable levels of star formation. The cooling time and entropy thresholds point to thermal instability in hot atmospheres fueling nebular emission and star formation (Nulsen 1986; Pizzolato \& Soker 2005). However, a convincing theoretical explanation of its value has proved elusive.

\subsection{Observational Inconsistency with $t_{c} / t_{f f}$ Threshold}

Several studies have argued that the cooling time and entropy thresholds are a consequence of thermal instabilities that develop in hot atmospheres when the ratio of $t_{\mathrm{c}} / t_{\mathrm{ff}}$ falls below 10 (McCourt et al. 2012; Sharma et al. 2012; Voit et al. 2015). If true, the ratio should more reliably forecast $\mathrm{H} \alpha$ emission than the cooling time or entropy index alone. We find that the $t_{\mathrm{c}} / t_{\mathrm{ff}} \lesssim 10$ criterion as applied in these studies is less reliable. For example, of more than 200 cluster cores studied by Cavagnolo et al. (2008), only five lying below the cooling time/entropy threshold failed to shine with $\mathrm{H} \alpha$ emission, an iconic example being Abell 2029, which we discuss in detail below. In contrast, only 10 of 43 systems in Voit \& Donahue (2015) with detectable $\mathrm{H} \alpha$ emission met the $t_{\mathrm{c}} / t_{\mathrm{ff}} \lesssim 10$ criterion.

We illustrate this point by calculating the $t_{\mathrm{c}} / t_{\mathrm{ff}}$ threshold as it applies to three iconic central cluster galaxies for which we plot the cooling time, free-fall time, and their ratio in Figure 1. The center and right panels of Figure 1 show the free-fall time profiles and $t_{\mathrm{c}} / t_{\mathrm{ff}}$ profiles, respectively, for each cluster. The cooling time profiles were derived from deprojected gas density profiles, which removes emission from hot gas at large atmospheric distances seen in projection. The free-fall times were estimated for Abell 2029 and MS 0735+7421 assuming hydrostatic Navarro-Frenk-White (NFW) profile fits to the $\mathrm{X}$-ray data beyond an altitude of about $30 \mathrm{kpc}$. Those profiles were then grafted to isothermal profiles within $30 \mathrm{kpc}$ whose 
masses are anchored to the stellar mass, which matches their stellar velocity dispersion profiles. The M87 data were taken from Russell et al. (2015), who adopted the NFW2 profile from Romanowsky \& Kochanek (2001). A complete discussion of our methodology and results for a large sample of clusters will be presented in Hogan et al. (2016, in preparation) and Pulido et al. (2016 in preparation).

We use these three iconic examples to illustrate a general trend. The central cooling times for all three objects fall near to or below the cooling time threshold of $\sim 5 \times 10^{8} \mathrm{yr}$, indicated by a dashed line in the left panel of Figure 1. M87 (Sparks et al. 2004) and MS 0735+7421 (Donahue et al. 1992) shine in nebular emission within 5-30 kpc of their nuclei, as expected based on their short central cooling times. $\mathrm{H} \alpha$ emission in central galaxies is associated with the presence of molecular clouds (Edge 2001). The third cluster, Abell 2029, has revealed no appreciable $\mathrm{H} \alpha$ emission (McDonald et al. 2010), [O II] $\lambda 3727$ emission, or star formation (McNamara \& O'Connell 1989) in its central galaxy, despite falling well below the cooling time threshold. All three lie well above $t_{\mathrm{c}} / t_{\mathrm{ff}} \lesssim 10$. By this criterion, shine in $\mathrm{H} \alpha$ emission, yet two do. At the same time, the cooling time threshold predicts that all three should shine with $\mathrm{H} \alpha$ emission, yet Abell 2029 does not. Something is awry. We suggest that new and interesting physics is needed to solve the problem. A more detailed description of these objects is given in Appendices A, B, and C.

Regardless of hosting AGNs spanning 4 decades of radiomechanical power, the cooling profiles for all three are remarkably similar. Due to their larger distances, the profiles for Abell $2029 \quad(z=0.077)$ and MS 0735+7421 $(z=0.216)$ are unresolved below a few kiloparsecs. Nevertheless, M87's cooling times at 2 and $6 \mathrm{kpc}$ are similar to those of Abell 2029 and MS 0735+7421 at similar altitudes. Based on their similar shapes, it would be tempting to suggest that the cooling time profiles for Abell 2029 and MS 0735+7421 continue to decline into the nucleus despite the vast differences in AGN mechanical power between the three objects.

The key point is, despite short central cooling times, the ratios of cooling time to free-fall time all lie well above 10 . Therefore, all should be thermally stable and devoid of $\mathrm{H} \alpha$ emission, yet two are not. McCourt et al. (2012) suggested that an upward departure from the $t_{\mathrm{c}} / t_{\mathrm{ff}} \lesssim 10$ criterion in systems with bright $\mathrm{H} \alpha$ emission, such as M87 and MS 0735+7421, may be a consequence of a temporary decrease in central gas density in response to AGN heating. This explanation cannot be excluded out of hand given the scatter in the central cooling time profiles of clusters (Panagoulia et al. 2014). However, that the cooling profiles in Figure 1 are so similar, despite ongoing AGN activity in both M87 and MS 0735+7421, indicates that a dramatic increase in AGN power need not lead to a dramatic upward response in atmospheric cooling time, the radial run of $t_{\mathrm{c}} / t_{\mathrm{ff}}$, or central gas density.

This point is further illustrated in Figure 2, where we plot the radial run of gas density in the hot atmospheres of the three objects discussed here. In addition, we have included Abell 1835 , which hosts one of the largest reservoirs of molecular gas $\left(5 \times 10^{10} M_{\odot}\right)$ and one of the highest star formation rates ( $\sim 200 M_{\odot} \mathrm{yr}^{-1}$ ) known (McNamara et al. 2006, 2014). Figures 1 and 2 illustrate the surprisingly small variation in both gas density and cooling time of the hot atmospheres, despite an enormous range of AGN power. The objects shown in Figure 2 span 5 decades in AGN energy and nearly

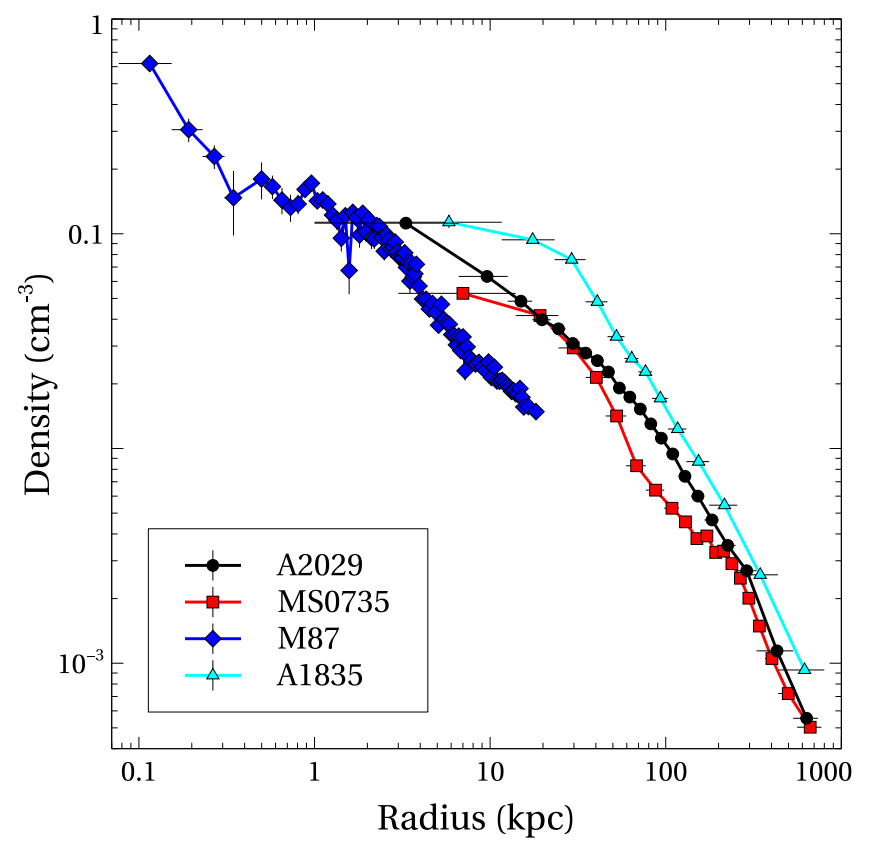

Figure 2. Radial dependence of electron density for the three objects presented in Figure 1. We have included Abell 1835 as an example galaxy with a large molecular gas mass of $5 \times 10^{10} M_{\odot}$ and a star formation rate approaching $200 M_{\odot} \mathrm{yr}^{-1}$ (McNamara et al. 2006). The outburst energies determined from cavity and shock front measurements are as follows: M87, $5 \times 10^{57} \mathrm{erg}$ (Forman et al. 2016); MS 0735+7421, $9 \times 10^{61} \mathrm{erg}$ (Vantyghem et al. 2014); Abell 1835, $4 \times 10^{59}$ erg (McNamara et al. 2006); and Abell 2029, no detectible shocks or cavities. Despite having experienced AGN outbursts spanning 5 decades in energy over the past several tens of megayears, their gas densities at $10 \mathrm{kpc}$ vary by only a factor of 5 and their cooling times at $10 \mathrm{kpc}$ are nearly identical.

4 decades in molecular gas mass. Yet the variation in their gas densities at $10 \mathrm{kpc}$, which is where most studies find a minimum value in $t_{\mathrm{c}} / t_{\mathrm{ff}}$, lie in the narrow range of $(2-10) \times 10^{-2} \mathrm{~cm}^{-3}$. Furthermore, their cooling times at $10 \mathrm{kpc}$ are nearly identical. Despite vast differences in their star formation rates, their molecular gas masses, and, most importantly, their AGN power, their central X-ray gas densities and cooling times are remarkably steady, showing little evidence of cycling indicated in precipitation models. Evidently AGNs contribute little to the scatter in central cooling times found by Panagoulia et al. (2014), but instead probably reflect variations in their halo masses. The resilience of the central gas density and central cooling time to powerful AGN outbursts is, in fact, a key feature of hot atmospheres stabilized by continual and gentle AGN feedback (McNamara \& Nulsen 2012).

This is not to say that all clusters with multiphase gas fail the $t_{\mathrm{c}} / t_{\mathrm{ff}} \lesssim 10$ instability criterion. But we find that systems that obey the criterion do so because their central cooling times are short and not because free-fall times are long. Among the several studies that have examined this criterion (Sharma et al. 2012; Voit \& Donahue 2015; Voit et al. 2015; Loubser et al. 2016), all did so by calculating the ratio of the average cooling time in radial bins and dividing by an estimate of the free-fall time from a given altitude using a similar approach to ours. These studies assumed that thermal instability ensues if and where the minimum value of the $t_{\mathrm{c}} / t_{\mathrm{ff}}$ profile falls below 10. Minima are usually found at a radius of about $10 \mathrm{kpc}$, 


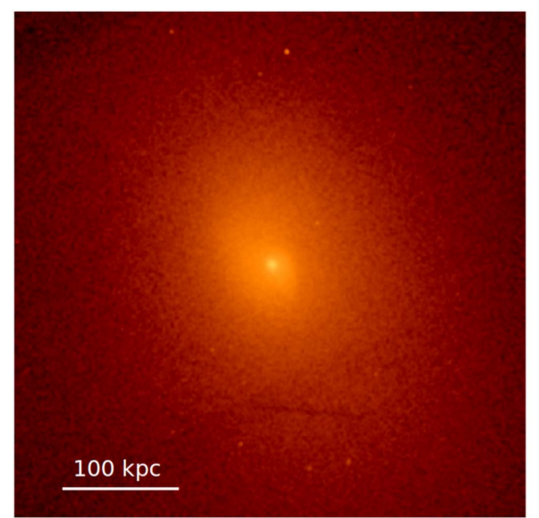

Abell 2029

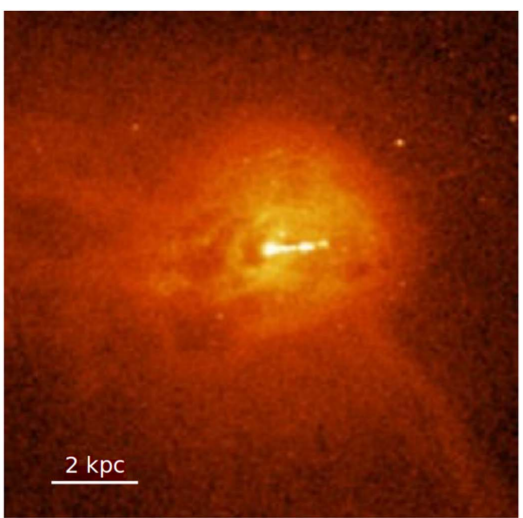

M87

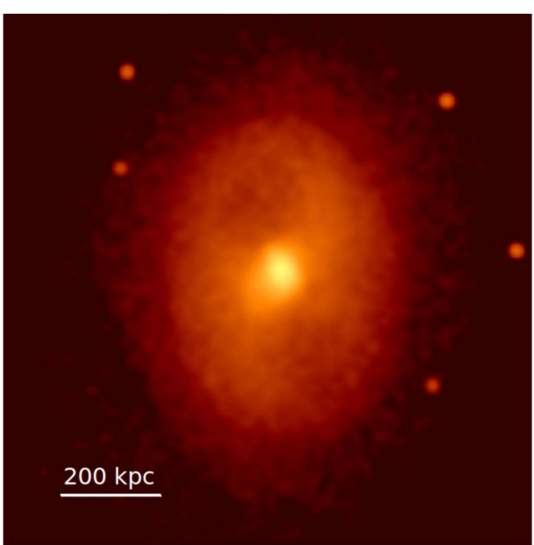

MS0735+7421

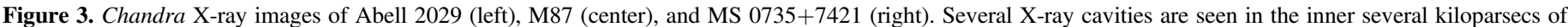

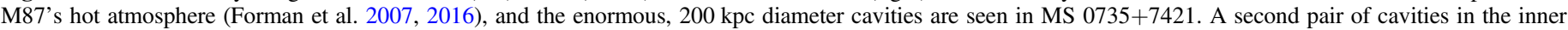

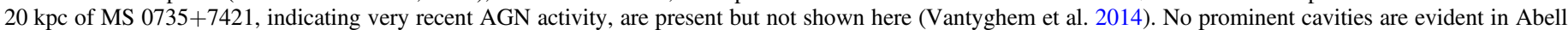
2029's hot atmosphere (Paterno-Mahler et al. 2013).

consistent with Figure 1. Their measured locations depend on several physical and nonphysical effects, including the relative slopes of the gas and free-fall time profiles and instrumental resolution. The largest uncertainty concerns the value of the free-fall time, as the acceleration is difficult to measure using standard techniques: stellar velocity dispersions are in short supply, and hydrostatic mass measurements are difficult to measure using standard techniques. The limited number of available velocity dispersions led Voit et al. (2015), understandably, to calculate the free-fall time adopting a velocity dispersion floor of $\sigma=250 \mathrm{~km} \mathrm{~s}^{-1}$. While reasonable on average, this assumption biases $t_{\mathrm{c}} / t_{\mathrm{ff}}$ artificially low as stellar velocity dispersions in central cluster galaxies often lie well above $300 \mathrm{~km} \mathrm{~s}^{-1}$. For example, M87 and Abell 2029, with velocity dispersions of $\sigma \sim 340 \mathrm{~km} \mathrm{~s}^{-1}$ (Gebhardt et al. 2011) and $\sigma \sim 400 \mathrm{~km} \mathrm{~s}^{-1}$ (Fisher et al. 1995), respectively, drive $t_{\mathrm{c}} / t_{\mathrm{ff}}$ well above 10 (Figure 1 ).

That central cooling time is driving the ratio is evident in Loubser et al. (2015), who studied star formation in a sample of 18 central galaxies, four of which are forming stars. The central cooling times of all four star formers lie below $0.5 \mathrm{Gyr}$, and their ratios of cooling time to free-fall time are claimed to lie below 10 , i.e., they apparently obey both the $t_{\mathrm{c}} \lesssim 5 \times 10^{8} \mathrm{yr}$ and $t_{\mathrm{c}} / t_{\mathrm{ff}} \lesssim 10$ criteria. The central cooling times and ratios of cooling time to free-fall time for the remaining red central galaxies, which are largely devoid of star formation, exceed 1 Gyr and 10, respectively. Thus, these objects are consistent with both criteria. However, the free-fall times for the short and long cooling time systems are consistent with a single value with small dispersion. The average free-fall time for the star-forming systems and dormant systems are $\left\langle t_{\mathrm{ff}}\right\rangle=0.064 \pm 0.016 \mathrm{Gyr}$ and $\left\langle t_{\mathrm{ff}}\right\rangle=0.055 \pm 0.014$, respectively. Therefore, $t_{\mathrm{c}} / t_{\mathrm{ff}}$ in this study, as in others, is insensitive to the free-fall time and is governed entirely by the cooling time, $t_{\mathrm{c}}$. We performed an analysis for Voit \& Donahue (2015) and found, similarly to Loubser et al. (2015), that dividing by the free-fall time only increases the scatter in the fundamental relationships between central cooling time, entropy, $\mathrm{H} \alpha$ emission (Cavagnolo et al. 2008), and star formation (Rafferty et al. 2008). Therefore, the $t_{\mathrm{c}} / t_{\mathrm{ff}} \lesssim 10$ criterion, as it has been applied, does not indicate the onset of cooling instabilities.

\subsection{Comparison between Observation and Simulation}

Three-dimensional, high-resolution simulations of the effects of AGN feedback on cooling atmospheres have offered new insights into AGN feedback. For example, Li \& Bryan (2014) and Li et al. (2015) modeled the response of X-ray atmospheres to AGN feedback over a period of several gigayears using an adaptive mesh refinement code, while Prasad et al. (2015) used two- and three-dimensional hydrodynamic simulations. Despite significant differences in approach, their model predictions are broadly similar to each other. In these simulations, hot atmospheres experience large swings in gas density, cooling time, molecular gas mass, and star formation rate in response to AGN power output over a span of several gigayears. The AGN power variations and star formation rates roughly correlate with rising and falling levels of molecular gas cooling from hot atmospheres. For example, Li et al. (2015) found swings in the minimum value of the cooling time and minimum value of $t_{\mathrm{c}} / t_{\mathrm{ff}}$ that vary by two orders of magnitude and factors of 25 , respectively, as the atmosphere breaths in response to variations in AGN power. Prasad et al. (2015) found similarly large amplitude swings in minimum $t_{\mathrm{c}} / t_{\mathrm{ff}}$ and jet power. The molecular gas mass in these models likewise varies by three to four orders of magnitude as it is consumed by star formation. In the Li et al. (2015) model, the molecular gas levels peak when the black hole, and presumably the radio $\mathrm{AGN}$, are at maximum accretion and power, respectively. The star formation rate, jet power, and molecular gas mass all move roughly together, albeit with a lag in time, over the several-gigayear simulations. As the cold gas is consumed by star formation, the jet power diminishes as its fuel supply subsides. Declining jet power causes the atmosphere to contract; the atmospheric gas density rises, causing $t_{\mathrm{c}}$ to drop as $t_{\mathrm{c}} / t_{\mathrm{ff}}$ approaches a minimum near unity; and the cooling cycle begins anew.

While these models capture the quasi-periodic nature of AGN feedback, we are unable to match the observed minimum values of $t_{\mathrm{c}} / t_{\mathrm{ff}} \simeq 20$ for MS 0735, M87, and Abell 2029 to the predicted minimum cooling time, jet power, molecular gas mass, and star formation rates at any time during the several gigayears spanning the models. For their minimum values of $t_{\mathrm{c}} / t_{\mathrm{ff}}$, the models generally predict star formation rates, molecular gas, and cooling times at levels exceeding 
those observed. Only Abell 1835, for which we find a minimum $t_{\mathrm{c}} / t_{\mathrm{ff}} \simeq 10$, may correspond to an acceptable solution at $\sim 4-5$ Gyr in the models of Li et al. (2015) and Prasad et al. (2015), as molecular gas builds up at late times.

Observational trends with molecular gas masses (Edge 2001) may offer additional insight. For example, no clear trend between total molecular gas mass and AGN power is found in central cluster galaxies (McNamara et al. 2011). The relationship between molecular gas mass and jet power reveals a 3decade scatter in both variables, superposed, perhaps, on a weak trend. Our main point is that high molecular gas masses do not necessarily lead to powerful AGN activity, at least when considering only central cluster galaxies (this may not be true for lower jet power elliptical galaxies). This point is clearly illustrated by the enigmatic MS 0735+7421. At $\sim 10^{46} \mathrm{erg} \mathrm{s}^{-1}$ over the past few hundred megayears, MS $0735+7421$ is the most energetic AGN outburst known. More importantly, its central atmospheric gas density at $10 \mathrm{kpc}$ is similar to others with vastly lower AGN power, and its molecular gas mass lies well below much weaker AGNs. Prasad et al. (2015) have argued, using their model, that the large scatter between molecular gas mass and jet power arises because the molecular gas is locked up in kiloparsec-scale disks (e.g., Gaspari et al. 2012) that are unable to fuel the central black hole. Whether this is generally true is not clear. ALMA observations, which are in short supply, would be required to resolve the molecular gas and to test the molecular disk hypothesis. The main difficulty comparing observation to the models is that we are unable to identify a reliable observational marker of the time-evolutionary state of these systems $\left(t_{\mathrm{c}} / t_{\mathrm{ff}}\right.$ is unsuited). Once we do, ALMA will in principle test whether objects with the highest molecular gas masses are evolutionarily advanced.

The crux of the problem, in our view, is that we do not observe the large-amplitude swings in atmospheric gas density (Figure 2) or cooling time (Figure 1) that correspond to atmospheric "overheating" implied by simulation (Gaspari et al. 2012; Li \& Bryan 2014; Li et al. 2015). Nor do we find that $t_{\mathrm{c}} / t_{\mathrm{ff}} \lesssim 10$ corresponds to the onset of cooling instability. However, Li \& Bryan (2014) and Li et al. (2015) pointed out that cooling is enhanced in their simulation by turbulence and when AGNs lifted hot gas to higher altitudes (McNamara et al. 2014; Voit \& Donahue 2015). Both processes tend to increase the infall time of the cooling gas driving the local value $t_{\mathrm{c}} / t_{\mathrm{ff}}$ toward unity. This is an important result that we believe is key to understanding the thermal instability of hot atmospheres.

\section{A MECHANISM FOR STIMULATED FEEDBACK}

We propose an alternative mechanism for driving cooling instabilities in hot atmospheres surrounding elliptical galaxies (Werner et al. 2014) and central cluster galaxies (Edge 2001; O'Dea et al. 2008) that incorporates essential physics of the McCourt et al. (2012) and Sharma et al. (2012) model and the precipitation model of Voit et al. (2015), but is motivated primarily by observation. The mechanism, which we refer to as stimulated feedback, simply posits that molecular clouds condense from cool, low-entropy gas lifted in the wakes of buoyantly rising X-ray bubbles to an altitude where the timescale for the clouds to return to their equilibrium position in the central galaxy approaches their radiative cooling time, i.e., $t_{\mathrm{c}} / t_{I} \lesssim 1$. Here $t_{\mathrm{I}}$ is the infall time for thermally unstable clouds whose value depends on factors that may vary within and among systems. The dynamics of a cloud are determined by, at least, the competing effects of gravity and drag (e.g., Cowie et al. 1980; Nulsen 1986; Pizzolato \& Soker 2005), and flow in the hot gas. If the terminal speed of the cloud is smaller than typical infall speeds, it can be lifted and pushed around by hot gas flows, and its infall speed will not generally exceed its terminal speed, $\simeq v_{K} \sqrt{r \delta \rho /\left(R \rho_{e}\right)}$, where $R$ is the distance to the cluster center, $\rho_{e}$ is the ambient gas density, the cloud density is $\rho_{e}+\delta \rho$, and its depth is $r$. If the terminal speed of the cloud is greater than typical infall speeds, the cloud will free-fall. Thus, the infall speed of a cooling cloud is generally limited to the lesser of its terminal speed and the free-fall speed. Angular momentum, magnetic, and other stresses might further complicate cloud dynamics, but they do not alter this conclusion.

Molecular cloud speeds observed with ALMA (David et al. 2014; McNamara et al. 2014; Russell et al. 2016) indicate that the infall timescale is likely a few times longer than $t_{\mathrm{ff}}$. This mechanism is motivated by ALMA and Chandra X-ray observations of molecular and atomic gas in central galaxies indicating hot and cold gas flows behind buoyantly rising X-ray cavities, and by insights from numerical simulations that closely resemble observed molecular cloud morphologies (Li \& Bryan 2014; Brighenti et al. 2015). In the next subsection we describe the observational indications for this mechanism.

\subsection{Driving Molecular Gas Flows by AGNs in Clusters and Groups}

Studies of the Perseus Cluster have revealed an association between molecular clouds, $\mathrm{H} \alpha$ filaments, and buoyantly rising X-ray bubbles (Salomé et al. 2011). Tendrils of molecular gas have apparently been lifted tens of kiloparsecs in altitude from NGC 1275 into the Perseus Cluster, with velocity fields consistent with inflow and/or outflow. ALMA observations of several other central cluster galaxies (including Abell 1835 and PKS 0745-191) have since revealed similar molecular cloud complexes lying beneath buoyantly rising X-ray cavities. Clouds are seen in thin filaments, with velocity fields and locations that further suggest a close relationship between X-ray bubbles and molecular gas (McNamara et al. 2014; Russell et al. 2016).

The Cycle 0 observations of Abell 1835 (McNamara et al. 2014) revealed two velocity components: a fast component of $10^{10} M_{\odot}$ of molecular clouds traveling with projected speeds of 200-500 $\mathrm{km} \mathrm{s}^{-1}$, and a slowly moving, $4 \times 10^{10} M_{\odot}$ component of molecular gas. The fast component lies beneath buoyantly rising X-ray cavities at altitudes of 5-10 kpc, while the slow component lies at the center of the galaxy associated with $\sim 200 M_{\odot} \mathrm{yr}^{-1}$ of star formation. The fast clouds are likely an outflow propelled by the rising X-ray bubbles. However, it is unclear how the molecular clouds are accelerated. Are molecular clouds themselves lifted and accelerated by the bubbles and jets, or is molecular gas condensing from hot, keV gas lifted in the bubbles' wakes? The answer may be "both." Acceleration is an issue not just in clusters but in active galaxies in general (Cicone et al. 2014; Morganti et al. 2015), where the molecular outflows are thought to be driven by jets (e.g., Nesvadba et al. 2008; Wagner \& Bicknell 2011) and winds.

The difficulty in lifting $10^{10} M_{\odot}$ of molecular clouds by jets and bubbles has been discussed in detail by Russell et al. (2016), McNamara et al. (2014), and David et al. (2014), who 
found in all cases that AGNs release enough energy to account for the kinetic energy in molecular gas flows. However, whether low-density jets have sufficient momentum to accelerate molecular clouds, whose densities exceed jet densities by four or five orders of magnitude, is unclear.

The total momentum flux (force) available from a kineticenergy-dominated jet of power $P_{j}$ is $\left(\Gamma_{j}+1\right) P_{j} /\left(\Gamma_{j} v_{j}\right)$, where $v_{j}$ is the jet speed and $\Gamma_{j}$ is the corresponding Lorentz factor. The buoyant force due to a bubble is $F_{B}=\rho_{e} g V$, where $g=v_{K}^{2} / R$ is the acceleration due to gravity. Estimating the volume as $V=H /(4 p)=P_{j} t_{j} /(4 p) \quad$ gives $\quad F_{B}=\left(P_{j} / v_{B}\right)\left[v_{K}^{2} /\left(4 p / \rho_{e}\right)\right]$, where the mean speed of the bubble as it formed is $v_{B}=R / t_{j}$, and $H$ is the bubble's enthalpy. The factor in square brackets is of order unity, and the mean speed of the bubble is comparable to the sound speed. Therefore, the buoyant force exceeds the jet ram pressure unless the flow speed of the jet is transonic relative to the atmosphere or slower. The small cross sections of jets make them even less effective at lifting.

Bubble buoyancy is generally more effective at lifting than the ram pressure of the jet that inflated it. The lifting ability of radio bubbles in general is limited by Archimedes's principle, which prohibits them from lifting more weight than they displace. The displaced mass in Abell 1835 is uncomfortably close to the $\sim 10^{10} M_{\odot}$ of molecular gas flowing behind the bubbles (McNamara et al. 2014), while in PKS 0745-191 the bubbles displace roughly 10 times the molecular gas mass (Russell et al. 2016). However, David et al. (2014) found that the molecular gas mass substantially exceeds the displaced mass in NGC 5044. Therefore, bubbles may be able to lift the molecular gas in Abell 1835 and PKS 0745-191, while NGC 5044's bubbles would be unable to do so.

Additional clues may be found from the radio sources themselves. Observations of the Abell 2597 and Abell 1795 central galaxies have shown that their radio jets bend by roughly $90^{\circ}$ at the locations of molecular clouds and knots of star formation (McNamara et al. 1996; Salomé \& Combes 2004, Tremblay et al. 2016, in preparation). The sharp bending suggests that a collision between the ensemble of molecular clouds and jets halted the jets' forward momenta (McNamara et al. 1996). Therefore, the jets are unlikely to be accelerating the molecular clouds appreciably, at least over the time the jets have been in contact with the molecular clouds. In fact, the molecular clouds in Abell 2597 are moving below the circular speed at their radius (Tremblay et al. 2016, in preparation) and should then be falling in rather than being driven out.

In summary, observation indicates that some molecular gas is lifted directly by radio jets and bubbles. But the often large molecular gas masses relative to the hot gas mass displaced by $\mathrm{X}$-ray bubbles suggests that some or most is lifted in the hot phase. Lifting hot, volume-filling gas behind the bubbles is easier, and the coolest gas may be able to condense into molecular clouds on the same timescales in which bubbles rise to their observed locations (McNamara et al. 2014; Russell et al. 2016).

\subsection{Does Molecular Gas Condense from Hot Outflows?}

Chandra has revealed columns of high-metallicity gas along and behind X-ray bubbles in clusters. Gas with high metallicity approaching and sometimes exceeding the solar value, enriched by stellar evolution, accumulates around central galaxies. The metal-rich columns of gas extending tens to hundreds of kiloparsecs in elevation are thought to trace hot flows lifted outward by radio bubbles (Simionescu et al. 2008; Kirkpatrick et al. 2009, 2011; Werner et al. 2010; Werner et al. (2011)). This phenomenon is also seen in hydrodynamic simulations where metal-enriched gas in the central galaxy is propelled outward in the updraft of rising bubbles (Pope et al. 2010; Gaspari et al. 2011). Estimated flow rates of several tens of solar masses per year would be sufficient to account for the observed molecular gas masses. The altitudes achieved are roughly proportional to the square root of the jet power (Morsony et al. 2010; Kirkpatrick \& McNamara 2015) and are on the order of tens of kiloparsecs for typical cluster AGNs, and several hundred kiloparsecs in the most powerful systems such as MS 0735+7421 and Hydra A (Simionescu et al. 2008; Kirkpatrick et al. 2011). Therefore, the lifting of hot gas by radio bubbles would be at least a plausible source of fuel for molecular cloud condensations at high altitudes.

Among the puzzling results from early ALMA observations of clusters are the surprisingly low radial velocities and velocity widths of the molecular gas. In response to the slow inner cloud velocities in Abell 1835, we suggested the mundane and unlikely possibility that the slow (radially) moving nuclear clouds are rotationally supported in a disk viewed in the plane of the sky (McNamara et al. 2014). While this interpretation may apply to Abell 1835 itself, cloud velocities lying well below the stellar velocity dispersion or circular speeds have now been seen in several systems (David et al. 2014; Russell et al. 2015, 2016, Tremblay et al. 2016, in preparation), indicating a phenomenon unrelated to rotational support or orientation effects. Traveling well below their expected gravitational speeds, either the clouds have had no time to relax in their gravitational potential wells and thus are surprisingly young, or they are pinned by magnetic fields or dynamic pressure to the hot gas from which they cooled, or some combination (Russell et al. 2016). Regardless of the cause, if molecular cloud velocities are representative of the speeds of condensing clouds, their velocities are several times lower than their hosts' stellar velocity dispersions and below their expected free-fall speeds.

\subsection{Stimulated Cooling at High Altitudes}

Following the arguments of Nulsen (1986), lowentropy $(\lesssim 1 \mathrm{keV})$ gas should condense into molecular clouds when lifted to an altitude where the ratio of its cooling time to infall time, $t_{\mathrm{c}} / t_{\mathrm{I}}$, approaches unity. Here, the infall time can be longer than the free-fall time, as indicated by ALMA observations. We postulate that systems with short central cooling times $t_{\mathrm{c}} \lesssim 5 \times 10^{8} \mathrm{yr}$, yet lacking X-ray cavities powerful enough to lift low-entropy gas to altitudes where $t_{\mathrm{c}} / t_{\mathrm{I}} \sim 1$, remain thermally stable and thus do not shine with nebular emission.

For example, Abell 2029 is apparently thermally stable, i.e., $t_{\mathrm{c}} / t_{\mathrm{I}}>1$ throughout its hot atmosphere. Figure 1 shows that the mean atmospheric cooling time within $10 \mathrm{kpc}$ is $t_{\mathrm{c}}=(3-5) \times 10^{8} \mathrm{yr}$. Over this volume, $t_{\mathrm{c}} / t_{\mathrm{ff}}=20-30$ and its atmosphere remains thermally stable, despite its short central cooling time. The atmosphere will become unstable where $t_{\mathrm{c}} / t_{\mathrm{ff}} \lesssim 1$. Its AGN must then lift hot gas from the inner $10 \mathrm{kpc}$ to altitudes between $\sim 280$ and $400 \mathrm{kpc}$, where the free-fall time is approximately equal to the cooling time of the low-entropy gas lifted from within $10 \mathrm{kpc}$. Relaxing the thermal instability criterion to $t_{\mathrm{c}} / t_{\mathrm{ff}} \lesssim 10$ implies lifting altitudes between 15 and $30 \mathrm{kpc}$. Upon lifting the cooler, denser central gas into the 

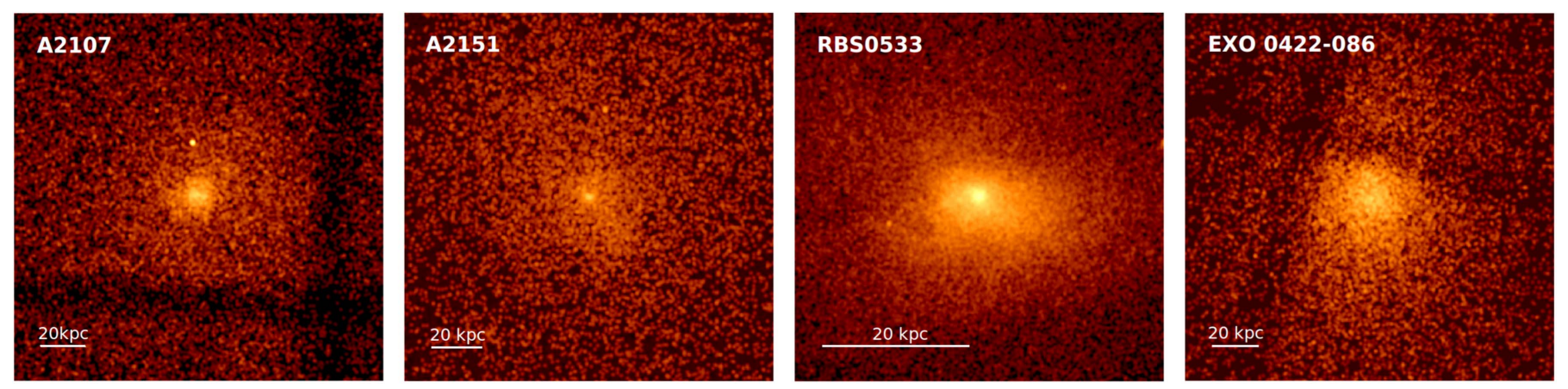

Figure 4. Chandra X-ray postage stamp images of the central 200 by 200". of the "spoiler" clusters from Cavagnolo et al. (2008): Abell 2107, Abell 2151, RBS 0533, EX 00422-086. The fifth spoiler is Abell 2029, shown in Figure 2. None reveal prominent radio bubbles.

lower-pressure atmosphere at higher altitudes, the lifted gas will expand and cool. Being denser than its surroundings, it should detach from the outflowing gas behind the bubble and fall back to the galaxy as it condenses into molecular clouds. If the cooling gas remains tethered to the surrounding atmosphere by magnetic fields or dynamical pressure, as ALMA observations suggest, its infall time, being governed by the terminal speed, would exceed the free-fall time. This would reduce the lifting altitude required to initiate cooling, such that $t_{\mathrm{c}} / t_{\mathrm{I}}$ approaches unity. In this picture, Abell 2029 fails to shine in $\mathrm{H} \alpha$ emission because its radio emission, despite being fairly powerful, lies within $25 \mathrm{kpc}$ of the nucleus (Paterno-Mahler et al. 2013). Abell 2029 has apparently not developed cavities capable of lifting low-entropy gas to the altitudes required to destabilize it (see Figure 3).

A similar analysis for MS $0735+7421$ gives similar figures. However, unlike Abell 2029, its powerful X-ray bubbles are lifting hot gas to altitudes upward of $300 \mathrm{kpc}$ (Kirkpatrick et al. 2011), well beyond the elevations required to stimulate cooling. As expected, its $\mathrm{H} \alpha$ emission extends $20-40 \mathrm{kpc}$ in the central galaxy with a luminosity among the highest known in a cluster (Donahue et al. 1992). The third example, M87, lying in a cooler atmosphere, will destabilize at lower altitudes and thus requires a lower-power AGN to stimulate cooling. Its average cooling time within a kiloparsec or so lies below $\sim 10^{8} \mathrm{yr}$. Therefore, following on the previous examples, its bubbles must lift this gas to altitudes of only $5-20 \mathrm{kpc}$ or so to initiate thermal instability. Shock fronts, bubbles (Forman et al. 2007), and metal-enriched gas columns (Simionescu et al. 2008) are observed in M87 to elevations exceeding $10 \mathrm{kpc}$, and nebular emission is observed within a similar volume (Sparks et al. 2004), which again is consistent with our model.

Perhaps the best example is the Perseus Cluster, which contains $\sim 10^{10} M_{\odot}$ of molecular gas centered on NGC 1275 and in filaments extending to altitudes of 30-50 kpc (Fabian et al. 2003; Salomé et al. 2011). The cooling time of its ambient gas exceeds the free-fall time by more than an order of magnitude and should be thermally stable as $t_{\mathrm{c}} / t_{\mathrm{ff}}>10$ at these elevations. However, the association between molecular gas, $\mathrm{H} \alpha$ filaments, and its system of X-ray bubbles is consistent with low-entropy gas lifted to altitudes where it can cool and return to fuel star formation in NGC 1275.

Finally, we have examined archival Chandra images of the five "spoilers" in Cavagnolo et al. (2008), those systems whose central atmospheric cooling times fall below the cooling time threshold yet lack detectable $\mathrm{H} \alpha$ emission. Like Abell 2029, and shown here in Figure 4, only one, RBS 0533, has revealed a possible cavity located approximately $10 \mathrm{kpc}$ to the southeast of its centroid, but otherwise no prominent cavity systems are seen (Abell 2029 is among the five). The archival images shown in Figure 4 have not been exposed deeply enough to exclude possible faint cavities at large radii. Nevertheless, until deeper exposures are obtained, the data in hand are consistent with our phenomenological model. Stimulated feedback is thus a viable and testable alternative to the conceptually important thermal instability (McCourt et al. 2012) and precipitation (Voit et al. 2015) models, which are inconsistent with observation.

\section{SUMMARY AND FINAL REMARKS}

Motivated primarily by new ALMA observations, we have proposed a new phenomenological model for the onset of thermal instabilities leading to nebular emission and star formation in giant galaxies. Molecular condensations form when low-entropy gas lying within a central galaxy is lifted to higher altitudes behind buoyantly rising X-ray bubbles inflated by radio AGNs. Lifting the low-entropy gas effectively increases its infall time, promoting condensation into molecular clouds in the bubbles' wakes. The surprisingly slow molecular cloud velocities found by ALMA indicate that the infall timescale, $t_{\mathrm{I}}$, can be significantly longer than the free-fall timescale. In this new picture, the rising $\mathrm{X}$-ray bubbles responsible for heating hot atmospheres simultaneously lift molecular gas and promote cooling in their wakes. This mechanism fuels steady feedback which we refer to as stimulated feedback. Molecular clouds eventually return to the central galaxy in a circulating flow that fuels star formation and the AGN itself. Once stimulated feedback commences, it naturally sustains itself, consistent with the prevalence of feedback in clusters and galaxies to large look-back times (Best et al. 2007; Ma et al. 2013; Hlavacek-Larrondo et al. 2015).

The amount of hot gas available to be lifted within the inner $10-20 \mathrm{kpc}$ of most clusters lies between several times $10^{9} M_{\odot}$ to several times $10^{10} M_{\odot}$, which would amply supply the observed levels of molecular gas in most galaxies (Edge 2001). In extreme instances, such as Abell 1835, where the molecular gas mass in the central galaxy exceeds the hot gas mass within a similar volume, the fuel supply must have accumulated from multiple AGN outbursts or have been augmented by other cooling channels. Our phenomenological model can be ruled out if it can be shown to be inconsistent with the cooling time and entropy thresholds. It must explain systems lying below the thresholds that lack $\mathrm{H} \alpha$ emission, which implies that their radio AGNs are too weak to lift gas to an altitude where it becomes 
thermally unstable. The model has the interesting property that once it gets started, it is potentially self-sustaining, which is an essential aspect of any feedback model. Because in stimulated feedback the jet must be able to lift the gas that eventually cools into molecular gas and stars to high altitudes, it may be more stable and less prone to overcooling that would lead to unrealistically high molecular gas masses and star formation rates seen in precipitation simulations.

Understanding how stimulated feedback leads to the precise values of the cooling time and entropy thresholds for the onset of $\mathrm{H} \alpha$ emission and star formation and determining what the value of $t_{\mathrm{c}} / t_{\mathrm{I}}$ must be to stimulate thermal instability are interesting challenges. That the cooling time threshold's value of $t_{\mathrm{c}} \sim 5 \times 10^{8} \mathrm{yr}$ is close to the maximum cycle duration for AGN feedback (Birzan et al. 2013; Vantyghem et al. 2014) is noteworthy. Furthermore, the lifting altitude at which the freefall time is roughly equal to the value of the cooling time threshold is several hundred kiloparsecs in the most massive clusters. This altitude is close to the highest lifting altitudes achieved by powerful AGNs in clusters (Kirkpatrick \& McNamara 2015). Therefore, the cooling time threshold may be set by the jet power itself.

It must also be understood why central galaxies bright with $\mathrm{H} \alpha$ emission that obey the cooling time threshold do not all have appreciable ongoing star formation. Those include the three objects highlighted here. We suggested that cooling instabilities in the star-forming galaxies have advanced to the point that they have accreted the critical surface density of molecular clouds required for stars to form. Finally, the question of what gets the mechanism started need not be a problem. An influx of gas, whether from the X-ray atmosphere or stripped from a passing galaxy or merger, could initiate it. These questions can be tested observationally and explored theoretically, and they may eventually overcome the problems with precipitation models.

B.R.M. acknowledges generous financial support from the Natural Sciences and Engineering Research Council of Canada and the Canadian Space Agency. H.R.R. and A.C.F. acknowledge support from ERC Advanced Grant 340442. We acknowledge helpful discussions with Mark Voit, Megan Donahue, Prateek Sharma, and the anonymous referee. This work was supported in part by Chandra Award Number G05$16134 \mathrm{X}$.

\section{APPENDIX A M87}

Owing to M87's proximity, we are able to follow its declining cooling time profile in Figure 1 from a value of $\sim 1 \mathrm{Gyr}$ at an altitude of $20 \mathrm{kpc}$ to $3 \times 10^{7} \mathrm{yr}$ within $200 \mathrm{pc}$ of the nucleus (Russell et al. 2015). The atmospheric cooling time remains near to or below $10^{8} \mathrm{yr}$ within $1 \mathrm{kpc}$, well below the cooling time threshold. Several X-ray cavities and a series of weak shock fronts lie within $10 \mathrm{kpc}$ of M87's nucleus associated with its radio source, indicating a total AGN power of $8 \times 10^{42} \mathrm{erg} \mathrm{s}^{-1}$ (Forman et al. 2007; Russell et al. 2013). M87 harbors bright nebular emission within $10 \mathrm{kpc}$, where the cooling time lies below $t_{\mathrm{c}}=6 \times 10^{8} \mathrm{yr}$, consistent with the cooling time threshold.

\section{APPENDIX B \\ MS $0735+7421$}

The MS 0735+7421 cluster's AGN, the most energetic known, has inflated enormous cavities, $200 \mathrm{kpc}$ in diameter (McNamara et al. 2005), with total energy expended by its cavities and surrounding shock fronts approaching $10^{62} \mathrm{erg}$ (Vantyghem et al. 2014). While the central galaxy shows no indication of star formation $\left(<0.5 M_{\odot} \mathrm{yr}^{-1}\right)$, it contains bright nebular emission indicating cooling, multiphase gas (Donahue et al. 1992). Its $\mathrm{H} \alpha$ nebula, with a luminosity of $\sim 10^{42} \mathrm{erg} \mathrm{s}^{-1}$ extending to $30 \mathrm{kpc}$ in altitude, is among the most luminous known in a galaxy cluster (Donahue et al. 1992). As expected, MS 0735+7421's atmospheric cooling time within $10 \mathrm{kpc}$ drops to $t_{\mathrm{c}}=5 \times 10^{8} \mathrm{yr}$, which lies close to the cooling time threshold.

\section{APPENDIX C \\ ABELL 2029}

We are able to measure Abell 2029's cooling time profile to an inner radius of $5 \mathrm{kpc}$, where its radiative cooling time approaches $2 \times 10^{8} \mathrm{yr}$, well below the cooling time threshold. In this respect, its X-ray atmosphere is similar to other clusters whose central galaxies are burgeoning with star formation fueled by reservoirs of upward of $10^{9} M_{\odot}$ of molecular gas, such as Abell 1795 and Abell 1835, yet it shows no sign of star formation or nebular emission (Johnstone et al. 1987; Johnstone \& Fabian 1988; McNamara \& O'Connell 1989). Abell 2029's central galaxy hosts a strong radio source, with a $1.4 \mathrm{GHz}$ luminosity of $L_{1.4} \sim 10^{42} \mathrm{erg} \mathrm{s}^{-1} \quad$ (Cavagnolo et al. 2008). An early claimed detection of weak X-ray cavities (Rafferty et al. 2006) is not confirmed in deeper X-ray data, although a cold front is visible in Figure 3. Apart from the cold front and some larger-scale structure, its atmosphere is relatively smooth (Paterno-Mahler et al. 2013).

\section{REFERENCES}

Alexander, D. M., Swinbank, A. M., Smail, I., McDermid, R., \& Nesvadba, N. P. H. 2010, MNRAS, 402, 2211

Arav, N., Moe, M., Costantini, E., et al. 2008, ApJ, 681, 954

Best, P. N., von der Linden, A., Kauffmann, G., Heckman, T. M., \& Kaiser, C. R. 2007, MNRAS, 379, 894

Bîrzan, L., Rafferty, D. A., McNamara, B. R., Wise, M. W., \& Nulsen, P. E. J. 2004, ApJ, 607, 800

Birzan, L., Rafferty, D. A., Nulsen, P. E. J., et al. 2013, AN, 334, 390

Bower, R. G., Benson, A. J., Malbon, R., et al. 2006, MNRAS, 370, 645

Brighenti, F., Mathews, W. G., \& Temi, P. 2015, ApJ, 802, 118

Cavagnolo, K. W., Donahue, M., Voit, G. M., \& Sun, M. 2008, ApJL, 683, L107

Cicone, C., Maiolino, R., Sturm, E., et al. 2014, A\&A, 562, A21

Cowie, L. L., Fabian, A. C., \& Nulsen, P. E. J. 1980, MNRAS, 191, 399

Cowie, L. L., Hu, E. M., Jenkins, E. B., \& York, D. G. 1983, ApJ, 272, 29

Croton, D. J., Springel, V., White, S. D. M., et al. 2006, MNRAS, 365, 11

David, L. P., Lim, J., Forman, W., et al. 2014, ApJ, 792, 94

Donahue, M., Stocke, J. T., \& Gioia, I. M. 1992, ApJ, 385, 49

Dunn, R. J. H., \& Fabian, A. C. 2008, MNRAS, 385, 757

Edge, A. C. 2001, MNRAS, 328, 762

Fabian, A. C. 2012, ARA\&A, 50, 455

Fabian, A. C., \& Sanders, J. S. 2007, in Eso Astrophysics Symp., Heating versus Cooling in Galaxies and Clusters of Galaxies, ed. H. Böhringer et al. (Berlin: Springer), 65

Fabian, A. C., Sanders, J. S., Crawford, C. S., et al. 2003, MNRAS, 344, L48

Feruglio, C., Maiolino, R., Piconcelli, E., et al. 2010, A\&A, 518, L155

Finoguenov, A., \& Jones, C. 2001, ApJL, 547, L107

Fisher, D., Illingworth, G., \& Franx, M. 1995, ApJ, 438, 539

Forman, W., Jones, C., Churazov, E., et al. 2007, ApJ, 665, 1057

Forman, W. R., Churazov, E., Jones, C., et al. 2016, BAAS, 227, 209.01 
Gaspari, M., Melioli, C., Brighenti, F., \& D'Ercole, A. 2011, MNRAS, 411,349

Gaspari, M., Ruszkowski, M., \& Sharma, P. 2012, ApJ, 746, 94

Gebhardt, K., Adams, J., Richstone, D., et al. 2011, ApJ, 729, 119

Heckman, T. M., Baum, S. A., van Breugel, W. J. M., \& McCarthy, P. 1989, ApJ, 338, 48

Hlavacek-Larrondo, J., McDonald, M., Benson, B. A., et al. 2015, ApJ, 805, 35

Hu, E. M., Cowie, L. L., \& Wang, Z. 1985, ApJS, 59, 447

Johnstone, R. M., \& Fabian, A. C. 1988, MNRAS, 233, 581

Johnstone, R. M., Fabian, A. C., \& Nulsen, P. E. J. 1987, MNRAS, 224, 75

Kirkpatrick, C. C., Gitti, M., Cavagnolo, K. W., et al. 2009, ApJL, 707, L69

Kirkpatrick, C. C., \& McNamara, B. R. 2015, MNRAS, 452, 4361

Kirkpatrick, C. C., McNamara, B. R., \& Cavagnolo, K. W. 2011, ApJL, 731, L23

Li, Y., \& Bryan, G. L. 2014, ApJ, 789, 54

Li, Y., Bryan, G. L., Ruszkowski, M., et al. 2015, ApJ, 811, 73

Loubser, S. I., Babul, A., Hoekstra, H., et al. 2016, MNRAS, 456, 1565

Ma, C.-J., McNamara, B. R., \& Nulsen, P. E. J. 2013, ApJ, 763, 63

McCourt, M., Sharma, P., Quataert, E., \& Parrish, I. J. 2012, MNRAS, 419, 3319

McDonald, M., Veilleux, S., Rupke, D. S. N., \& Mushotzky, R. 2010, ApJ, 721,1262

McNamara, B. R., \& Nulsen, P. E. J. 2007, ARA\&A, 45, 117

McNamara, B. R., \& Nulsen, P. E. J. 2012, NJPh, 14, 055023

McNamara, B. R., Nulsen, P. E. J., Wise, M. W., et al. 2005, Natur, 433, 45

McNamara, B. R., \& O'Connell, R. W. 1989, AJ, 98, 2018

McNamara, B. R., Rafferty, D. A., Bîrzan, L., et al. 2006, ApJ, 648, 164

McNamara, B. R., Rohanizadegan, M., \& Nulsen, P. E. J. 2011, ApJ, 727, 39

McNamara, B. R., Russell, H. R., Nulsen, P. E. J., et al. 2014, ApJ, 785, 44

McNamara, B. R., Wise, M., Sarazin, C. L., Jannuzi, B. T., \& Elston, R. 1996, ApJL, 466, L9

Morganti, R., Oosterloo, T., Oonk, J. B. R., Frieswijk, W., \& Tadhunter, C. 2015, A\&A, 580, A1

Morganti, R., Tadhunter, C. N., \& Oosterloo, T. A. 2005, A\&A, 444, L9

Morsony, B. J., Heinz, S., Brüggen, M., \& Ruszkowski, M. 2010, MNRAS, 407, 1277

Nesvadba, N. P. H., Lehnert, M. D., De Breuck, C., Gilbert, A. M., \& van Breugel, W. 2008, A\&A, 491, 407

Nulsen, P. E. J. 1986, MNRAS, 221, 377

O’Dea, C. P., Baum, S. A., Privon, G., et al. 2008, ApJ, 681, 1035

Panagoulia, E. K., Fabian, A. C., \& Sanders, J. S. 2014, MNRAS, 438, 2341
Paterno-Mahler, R., Blanton, E. L., Randall, S. W., \& Clarke, T. E. 2013, ApJ, 773,114

Pizzolato, F., \& Soker, N. 2005, ApJ, 632, 821

Pope, E. C. D., Babul, A., Pavlovski, G., Bower, R. G., \& Dotter, A. 2010, MNRAS, 406, 2023

Prasad, D., Sharma, P., \& Babul, A. 2015, ApJ, 811, 108

Rafferty, D. A., McNamara, B. R., \& Nulsen, P. E. J. 2008, ApJ, 687, 899

Rafferty, D. A., McNamara, B. R., Nulsen, P. E. J., \& Wise, M. W. 2006, ApJ, 652,216

Randall, S. W., Nulsen, P. E. J., Jones, C., et al. 2015, ApJ, 805, 112

Romanowsky, A. J., \& Kochanek, C. S. 2001, ApJ, 553, 722

Rupke, D., Veilleux, S., Kim, D.-C., et al. 2007, in ASP Conf. Ser. 373, The Central Engine of Active Galactic Nuclei, ed. L. C. Ho \& J.-W. Wang (San Francisco, CA: ASP), 525

Russell, H. R., Fabian, A. C., McNamara, B. R., \& Broderick, A. E. 2015, MNRAS, 451, 588

Russell, H. R., McNamara, B. R., Edge, A. C., et al. 2013, MNRAS, 432, 530

Russell, H. R., McNamara, B. R., Fabian, A. C., et al. 2016, MNRAS, 458, 3134

Salomé, P., \& Combes, F. 2003, A\&A, 412, 657

Salomé, P., \& Combes, F. 2004, A\&A, 415, L1

Salomé, P., Combes, F., Edge, A. C., et al. 2006, A\&A, 454, 437

Salomé, P., Combes, F., Revaz, Y., et al. 2008a, A\&A, 484, 317

Salomé, P., Combes, F., Revaz, Y., et al. 2011, A\&A, 531, A85

Salomé, P., Revaz, Y., Combes, F., et al. 2008b, A\&A, 483, 793

Sharma, P., McCourt, M., Quataert, E., \& Parrish, I. J. 2012, MNRAS, 420,3174

Simionescu, A., Werner, N., Finoguenov, A., Böhringer, H., \& Brüggen, M. 2008, A\&A, 482, 97

Sparks, W. B., Donahue, M., Jordán, A., Ferrarese, L., \& Côté, P. 2004, ApJ, 607,294

Vantyghem, A. N., McNamara, B. R., Russell, H. R., et al. 2014, MNRAS, 442, 3192

Voit, G. M., Cavagnolo, K. W., Donahue, M., et al. 2008, ApJL, 681, L5

Voit, G. M., \& Donahue, M. 2015, ApJL, 799, L1

Voit, G. M., Donahue, M., Bryan, G. L., \& McDonald, M. 2015, Natur, 519, 203

Wagner, A. Y., \& Bicknell, G. V. 2011, ApJ, 728, 29

Werner, N., Oonk, J. B. R., Sun, M., et al. 2014, MNRAS, 439, 2291

Werner, N., Simionescu, A., Million, E. T., et al. 2010, MNRAS, 407, 2063

Werner, N., Sun, M., Bagchi, J., et al. 2011, MNRAS, 415, 3369 